\title{
Sobre as pesquisas relacionadas ao ensino do efeito fotoelétrico ${ }^{+*}$
}

Ronivan Sousa da Silva ${ }^{1}$

Instituto Federal de Mato Grosso do Sul

Aquidauana - MS

Nádia Cristina Guimarães Errobidart ${ }^{2}$

Instituto de Física - Universidade Federal de Mato Grosso do Sul

Campo Grande - MS

\section{Resumo}

O número de pesquisas e os diferentes aspectos envolvidos no ensino de tópicos Física Moderna e Contemporânea (FMC) no ensino médio vêm crescendo de forma bastante considerável nos últimos anos, sendo necessário muitas vezes o levantamento e a categorização dos trabalhos publicados. Nesse sentido, nosso trabalho tem por objetivo apresentar o resultado de uma pesquisa bibliográfica relacionada exclusivamente ao ensino do efeito fotoelétrico, preferencialmente no ensino médio, realizada em periódicos nacionais e internacionais da área de ensino e educação. Tomamos como ponto de partida o trabalho de Ostermann e Moreira (2000) e seguimos até o final do ano de 2014. De acordo com nossos critérios de seleção, identificamos 41 artigos e os classificamos em oito categorias, a saber: (A) Fundamentação teórica e conceitual do efeito fotoelétrico; (B) Descrição de analogias e situações de contextualização do efeito fotoelétrico; (C) Aspectos de HFC com relação ao efeito fotoelétrico; (D) Propostas ou relatos do ensino do efeito fotoelétrico mediados por simulação computacional; (E) Propostas ou relatos do ensino do efeito fotoelétrico mediados por HFC; (F) Propostas ou relatos do ensino do efeito fotoelétrico mediados experimentação e $(G)$ Análise de livros didáticos ou ma-

\footnotetext{
${ }^{+}$On researches related to the teaching of the photoelectric effect

* Recebido: maio de 2015.

Aceito: agosto de 2015.

${ }^{1}$ E-mail: ronivan.silva@ifms.edu.br

2 E-mail: nadia.guimaraes@ufms.br
} 
nuais com relação à abordagem do efeito fotoelétrico. Os resultados indicam que maioria dos trabalhos publicados relatam propostas a serem utilizadas em sala de aula mediante utilização de simulação computacional ou experimentação ou aspectos de História e Filosofia da Ciência (HFC), porém, poucos pesquisadores efetivamente têm investigado o processo de construção dos conceitos físicos presentes no modelo corpuscular para a luz.

Palavras-chave: Física Moderna e Contemporânea; Revisão Bibliográfica; Efeito Fotoelétrico.

\begin{abstract}
The number of researches and different aspects involved in teaching topics of Modern and Contemporary Physics (MCF) in high school have grown quite considerably in recent years, often requiring the survey and categorization of published works. In this sense, our work aims to present the results of a literature review related exclusively to the teaching of the photoelectric effect, preferably in high school, from national and international journals in the area of Teaching and Education. We have taken the work of Ostermann and Moreira (2000) as a starting point and followed it until the end of 2014. According to our selection criteria, we have identified 41 articles and classified them into eight categories, namely: (A) Theoretical and conceptual basis of the photoelectric effect; (B) Description of analogies and situations of contextualization of the photoelectric effect; (C) HFC aspects regarding the photoelectric effect; (D) Proposals or teaching reports of the photoelectric effect mediated by computer simulation; (E) Proposals or teaching reports of the photoelectric effect mediated by HFC; $(F)$ proposals or teaching reports of the photoelectric effect mediated by experimentation and $(G)$ Analysis of textbooks or manual regarding the photoelectric effect approach. The results indicate that most published studies report proposals to be used in the classroom by using computer simulation, experimentation or aspects of History and Philosophy of Science (HPS), however, few researchers have effectively investigated the construction process of the physical concepts present in the corpuscular model of light.
\end{abstract}

Keywords: Modern and Contemporary Physics; Literature Review; Photoelectric Effect. 


\section{Introdução}

As últimas revisões bibliográficas sobre trabalhos envolvendo o ensino de Física Moderna e Contemporânea (FMC) evidenciaram um notável aumento no número de publicações desta linha de pesquisa (OSTERMANN; MOREIRA, 2000; GRECA; MOREIRA, 2001; e OSTERMANN; PEREIRA, 2009). As reflexões relacionadas às justificativas para a inserção de FMC no ambiente escolar, exploradas principalmente nos primeiros trabalhos, atualmente possibilitam aos pesquisadores direcionarem suas investigações para outros aspectos, tais como: construção de aparatos experimentais, elaboração de materiais didáticos e tecnologias da informação e comunicação, discussões sobre como inserir História e Filosofia da Ciência (HFC) ou questões de Ciência, Tecnologia, Sociedade e Ambiente (CTSA), entre outros.

De acordo com Ostermann e Pereira (2009), a maioria dos temas de pesquisa que abordam o ensino de FMC concentra-se em conceitos ligados à Mecânica Quântica (MQ), em seguida, têm-se vários trabalhos envolvendo conceitos da Teoria da Relatividade Restrita e, por fim, algumas pesquisas envolvendo tópicos como radiação, supercondutividade, física nuclear, física das partículas, entre outros.

Diante da ampla diversidade de tópicos de FMC estudados por esta linha de pesquisa, fizemos uma pesquisa bibliográfica relacionada exclusivamente ao ensino do efeito fotoelétrico, preferencialmente no ensino médio, realizada em periódicos nacionais e internacionais da área de ensino e educação. Tomamos como ponto de partida o trabalho de Ostermann e Moreira (2000) e seguimos até o final do ano de 2014. Nosso interesse por esse tema em particular está associado ao desenvolvimento de um projeto de pesquisa mais amplo, no qual procuramos investigar as contribuições e limitações de uma unidade de ensino potencialmente significativa, a qual é mediada por elementos de História e Filosofia da Ciência, relações Ciência-TecnologiaSociedade e uso de simulação computacional.

\section{Procedimentos metodológicos}

Inicialmente adotamos como recorte para nossa investigação os trabalhos publicados por Ostermann e Moreira (2000) e Ostermann e Pereira (2009). Particularmente com relação ao tema efeito fotoelétrico, no período compreendido entre 1987 a 2006, os autores identificaram estudos que abordavam as justificativas para sua inserção no ensino médio; tutoriais para construção de aparatos experimentais para laboratórios universitários; textos abordando aspectos teóricos e conceituais do fenômeno. Entretanto, apontaram a identificação de somente três trabalhos que relatavam propostas testadas em sala de aula (SILVA, 1993; GUNEL et al., 2006; KOVAČEVIĆ; DJORDJEVICH, 2006).

Com o objetivo de ampliarmos as revisões bibliográficas anteriormente discutidas até os dias atuais, seguimos os procedimentos de pesquisas bibliográficas sugeridos por Rosa (2013) como norteadores. Destacamos que esta metodologia é diferente daquela utilizada por Ostermann e Pereira (2009), pois os autores não fazem previamente uma seleção de palavras- 
chave, preferindo ler todos os artigos publicados nos periódicos. Esta metodologia também difere do trabalho de Ostermann e Moreira (2000) no que diz respeito à definição do escopo, pois os autores utilizaram artigos em revistas, livros didáticos, dissertações, teses, projetos e navegações pela internet, enquanto que nós restringimos nossas buscas apenas a periódicos nacionais e internacionais na área de ensino de ensino e educação.

Posto isto, Rosa (2013) sugere os seguintes passos para este tipo de pesquisa: i) definição de dez a 20 palavras-chave; ii) definição do escopo; iii) seleção do corpus e iv) análise. Considerando isso, foram definidas as seguintes palavras-chave: efeito fotoelétrico; natureza da luz; fóton; simulações computacionais de FMC; ensino de Mecânica Quântica (MQ); ensino de Física Quântica (FQ); Einstein; Hertz; quantum; analogias FMC e contextualização de FMC.

Partindo dos periódicos nos quais Ostermann e Moreira (2000) e Ostermann e Pereira (2009) encontraram trabalhos referentes ao ensino do efeito fotoelétrico, definimos os seguintes periódicos como fonte de busca (as siglas entre parênteses dizem respeito às abreviaturas que serão usadas no quadro 1):

1. Caderno Brasileiro de Ensino de Física (CBEF);

2. Revista Brasileira de Ensino de Física (RBEF);

3. A Física na Escola (FNE);

4. História da Ciência e do Ensino (HCE);

5. Physics Education (PE)

6. Science \& Education (SE)

7. American Journal of Physics (AJP)

8. American Institute of Physics (AIP)

Utilizamos o mecanismo de busca do próprio periódico para a sistematização das informações. Primeiramente, identificamos os artigos em que havia a ocorrência de pelo menos uma das 12 (doze) palavras-chaves em seus resumos. Nos casos em que houve certa dúvida sobre o enfoque do trabalho, foi feita uma leitura flutuante para identificar se o artigo tratava a respeito da abordagem do ensino do efeito fotoelétrico. Dentre as palavras-chave escolhidas, as que resultaram em maior número de trabalhos foram: efeito fotoelétrico; natureza da luz; fóton; simulações computacionais de FMC e Einstein.

No entanto, a partir das primeiras leituras, notamos que o tema escolhido apresentava um número muito grande de inter-relações com outros temas, por exemplo, reforma curricular, novo ensino médio, dualidade onda-partícula, entre outros. Este fato exigiu que nós adotássemos algum critério para seleção dos trabalhos a serem lidos na íntegra, para isto passamos a separar os trabalhos de acordo com o enfoque adotado pelos autores. Diante desta separação, notamos que poderíamos utilizar estes enfoques como categorias de classificação para os trabalhos aqui analisados. Assim, dividimos os trabalhos encontrados em oito categorias, a saber: (A) Fundamentação teórica e conceitual do efeito fotoelétrico; (B) Descrição de analogias e situações de contextualização do efeito fotoelétrico; (C) Aspectos de HFC com relação ao efeito da Silva, R. S. e Errobidart, N. C. G. 
fotoelétrico; (D) Propostas ou relatos do ensino do efeito fotoelétrico mediados por simulação computacional; (E) Propostas ou relatos do ensino do efeito fotoelétrico mediados por HFC; (F) Propostas ou relatos do ensino do efeito fotoelétrico mediados experimentação e (G) Análise nos livros didáticos ou manuais com relação à abordagem do efeito fotoelétrico.

No total, foram selecionados 41 artigos. O quadro 1 traz a distribuição desses trabalhos por periódicos em ordem crescente de datas de publicação.

Quadro 1: Distribuição dos artigos selecionados por revistas.

\begin{tabular}{|c|c|c|}
\hline $\begin{array}{l}\text { PERIÓDI- } \\
\text { COS }\end{array}$ & $\begin{array}{l}\text { N }^{\circ} \text { DE ARTI- } \\
\text { GOS }\end{array}$ & ARTIGOS \\
\hline CBEF & 11 & $\begin{array}{l}\text { Veit et al. (1987); Arruda e Filho (1991); Valadares e } \\
\text { Moreira (1998); Pinto e Zanetic (1999); Cavalcante e } \\
\text { Tavolaro (2001); Dionísio (2005); Coelho e Borges } \\
\text { (2010); Cardoso e Dickman (2012); Silva e Assis } \\
\text { (2012); Cavalcante et al. (2013); Cavalcante et al. } \\
\text { (2014) }\end{array}$ \\
\hline RBEF & 07 & $\begin{array}{l}\text { Gomes et al. (1996); Cavalcante et al. (2005); Filho et } \\
\text { al. (2006); Heckler } \text { et al. (2007); Sales et al. (2008); Pa- } \\
\text { ranhos } \text { et al. (2008); Alemany et al. (2013) }\end{array}$ \\
\hline FNE & 06 & $\begin{array}{l}\text { Medeiros (2005); Moreira (2005); Cavalcante et al. } \\
\text { (2002); Alves e Silva (2008); Silva e Martins (2009); } \\
\text { Neto et al. (2010) }\end{array}$ \\
\hline $\mathrm{HCE}$ & 01 & Mangili (2012) \\
\hline $\mathrm{PE}$ & 07 & $\begin{array}{l}\text { Jones (1991); Williams (2004); Whalley (2005); Ko- } \\
\text { vačević e Djordjevich (2006); Oon e Subramaniam } \\
\text { (2009a, 2009b); Sokolowski (2013) }\end{array}$ \\
\hline SE & 05 & $\begin{array}{l}\text { Kragh (1992); Stuewer (2006); Klassen (2009); Niaz et } \\
\text { al. (2010); Klassen et al (2011) }\end{array}$ \\
\hline AJP & 03 & $\begin{array}{l}\text { Steinberg et al. (1996); Oberem e Steinberg (1999); } \\
\text { McKagan et al. (2009) }\end{array}$ \\
\hline AIP & 01 & De Leone e Oberem (2003) \\
\hline TOTAL & 41 & \\
\hline
\end{tabular}

Fonte: Dados da Pesquisa.

Podemos observar no Quadro 1 que dos 41 artigos selecionados, 25 são nacionais e 16 internacionais. A maioria dos trabalhos foi publicada nas revistas Caderno Brasileiro de Ensino de Física (CBEF), Physics Education (PE), Revista Brasileira de Ensino de Física (RBEF) e A Física na Escola (FNE). Apresentamos abaixo uma descrição dos artigos selecionados de acordo com as categorias em que foram enquadrados. 


\section{Descrição e classificação dos trabalhos sobre o ensino do efeito fotoelétrico}

\section{Categoria A: Fundamentação teórica ou conceitual do efeito fotoelétrico (05 artigos)}

Na Inglaterra, analisando o ensino de FMC em um curso Nível A (curso extra oferecido para alunos acima de 16 anos, como forma de preparação para admissão em universidades) e na universidade, Jones (1991) critica a ênfase que é dada ao efeito fotoelétrico e à velha Mecânica Quântica. O autor questiona a abordagem estritamente histórica com que os temas são abordados, processo este que, segundo ele, em outras áreas, não é tão explicitamente observado.

Para o autor, o efeito fotoelétrico, apesar de ser considerado o núcleo central da introdução da FMC, parece ser apenas uma das consequências da análise de Einstein sobre a interação da matéria e o campo de radiação. Segundo o autor, este tópico não é a pedra angular da Mecânica Quântica e, portanto, construir o ensino "em cima” do conceito de fóton não é somente uma grande simplificação, mas também uma imagem falsa que pode ficar nas mentes dos estudantes. Para ele, a verdadeira pedra angular deveria ser o "Princípio da Incerteza de Heinseberg", pois este de fato seria um novo conceito para alunos acostumados com ensino da Física Clássica.

Gomes et al. (1996) apresentam uma proposta que vem sendo implementada na disciplina física moderna, do curso de graduação em Física (Licenciatura e Bacharelado) da Universidade Federal Fluminense, com vistas à melhoria da aprendizagem de fenômenos de interação da radiação com a matéria, mais especificamente os efeitos fotoelétrico, Compton e a produção/aniquilação de pares. O objetivo dos autores foi possibilitar uma contribuição na forma de um texto autoexplicativo, para professores e estudantes, de caráter complementar ao livro texto adotado.

Dionísio (2005) analisa o artigo intitulado "Sobre um ponto de vista heurístico a respeito da produção e transformação da luz", publicado por Albert Einstein em 1905. O autor pontua seu entendimento sobre como Einstein propôs, para o problema da radiação de corpo negro, uma solução diferente e independente da apresentada por Max Planck, cinco anos antes. Por fim, o autor sugere que os professores dos cursos de física moderna e autores de livros didáticos passem a usar a regra de Stokes, e não mais o efeito fotoelétrico, como o primeiro exemplo ilustrativo e a primeira evidência experimental da existência dos fótons, tal como fez o próprio Einstein em seu artigo original.

Medeiros (2005) relata um bate-papo fictício com Albert Einstein, num volume especial da revista que apresenta uma série de entrevistas com celebridades da Física. Neste texto, destinado a professores e alunos de ensino médio, o autor aborda os mistérios que assombravam a Física Clássica ao final do século XIX, período do nascimento da Física Quântica. A ideia central do texto é mostrar que estes mistérios advinham dos estudos envolvidos na radiação eletromagnética, isto é, com relação à sua natureza, propagação e interação com a matéria. Devido à amplitude do tema, o autor prefere não discutir neste texto como o problema da propagação da radiação levou ao nascimento da Teoria da Relatividade Restrita.

Moreira (2005) relata como o jovem Albert Einstein, trabalhando como técnico de $3^{\mathrm{a}}$ classe, em um escritório de patentes, em Berna (Suíça), publicou cinco trabalhos extremamente da Silva, R. S. e Errobidart, N. C. G. 
relevantes para história da Física. Os dois primeiros mostrariam como poderia ser demonstrada experimentalmente a realidade física dos átomos e moléculas, assunto que no início do século XX ainda era controverso. No terceiro artigo, Einstein propõe a ideia revolucionária de que a luz, sob certos aspectos, apresenta natureza corpuscular. E nos dois últimos, Einstein discute os postulados da Teoria da Relatividade Restrita, refutando o caráter absoluto atribuído, durante séculos, ao tempo e ao espaço.

Em síntese, os trabalhos classificados nessa categoria, buscam fornecer informações que minimizem ou até mesmo previnam a ocorrência de concepções errôneas ou reforço de mitos durante a abordagem deste tema em sala de aula. Por exemplo, é possível que muitos professores acreditem que o artigo de Einstein de 1905 foi primeiramente sobre a tentativa de explicar o efeito fotoelétrico, principalmente no que diz respeito às observações experimentais de Phillip Lenard em 1902, quando na verdade tratava-se de uma proposta revolucionária muito mais abrangente, a quantização da energia do campo eletromagnético.

\section{Categoria B: Descrição de analogias e situações de contextualização do efeito fotoelétrico (05 artigos)}

Com o objetivo de promover a contextualização dos conteúdos de Física no ensino médio, Valadares e Moreira (1998) apresentam sugestões conceituais e práticas de como abordar tópicos de FMC a partir de algumas aplicações tecnológicas presentes no cotidiano dos estudantes. Exploram o funcionamento de: dispositivos automáticos (portas de elevadores e esteiras de supermercados); sistemas de iluminação pública; fibras óticas; leitores de código de barras e LDR (resistor dependente da intensidade luminosa).

Williams (2004) propõe uma analogia (uma brincadeira ou jogo com viés lúdico) a ser realizada pelos alunos durante a apresentação do conceito de função trabalho de um metal. A brincadeira consiste de um chapéu (representando o átomo), bolas de tênis (representando elétrons) presas ao chapéu utilizando velcro e uma pistola de brinquedo que atira bolinhas (representando os fótons). $\mathrm{O}$ autor busca com isso reduzir o grau de abstração dos conceitos estudados no experimento do efeito fotoelétrico e fornecer uma estratégia de ensino que permita ao professor se afastar de uma descrição puramente teórica.

Whalley (2005) também propõe uma analogia para facilitar a aprendizagem do efeito fotoelétrico. Ele sugere pensar a função trabalho como a energia que um jogador precisa usar para fazer uma bola atravessar uma trave de um jogo de rugby. A energia do fóton seria a energia usada pelo jogador. O autor exemplifica sua analogia, destacando o fato de que 500 crianças (fótons de baixa energia) provavelmente nunca iriam obter êxito em sua jogada.

Kovačević e Djordjevich (2006) apresentaram uma analogia mecânica para o efeito fotoelétrico, assunto presente no currículo do último ano do ensino básico das escolas de Sérvia e Montenegro. Preocupados com o ensino desse tema, os autores propuseram um sistema de bolas rígidas e coloridas (onde cada cor corresponde a uma frequência do espectro luminoso) que deslizam sem atrito sobre uma rampa e colidem com outra bola rígida, lançando-a para fora do sistema. A energia inicial de cada fóton, entendido como bolas coloridas, a função trabalho 
do material foto-emissor e a energia cinética máxima dos elétrons emitidos, são analisadas em termos de diferença de altura em relação ao ponto mais baixo da rampa.

Alves e Silva (2008) abordam os detalhes do funcionamento das células fotovoltaicas e sugere uma atividade experimental de demonstração que possibilita o professor mostrar a transformação de energia solar em energia elétrica na sala de aula. As discussões sobre o funcionamento destas células são feitas basicamente sobre a física dos materiais semicondutores, por exemplo, o silício. Ao final, os autores apontam que esta atividade, quando utilizada em oficinas, sempre tem gerado um grande espanto quando os alunos observam um relógio digital funcionando com a energia transferida por um LED (diodo emissor de luz).

Em primeiro lugar, os trabalhos classificados nessa categoria fornecem subsídios para que o professor possa contextualizar, por meio de diferentes dispositivos tecnológicos, o ensino do efeito fotoelétrico. Em segundo, vale destacar a necessidade das analogias a serem utilizadas durante as aulas para este tema. Além disso, podemos destacar a observação de Klassen (2009) quando apontou que a maior dificuldade de aprendizagem das características do efeito fotoelétrico é identificada na interpretação da função trabalho na equação de Einstein.

\section{Categoria C: Aspectos de HFC com relação ao efeito fotoelétrico (07 artigos)}

Kragh (1992) argumenta que a relação entre Física e História da Física é intrinsicamente problemática, pois as lições a serem aprendidas da história são muitas vezes contraproducentes aos objetivos do ensino de ciências. Baseado na história do efeito fotoelétrico, como um caso de ensino para introdução da teoria quântica, o autor argumenta que o dilema entre a "verdadeira história" e a "utilidade didática" pode ser contornado focando alguns estudos de casos cuidadosamente selecionados.

Stuewer (2006) defende que a apresentação de surpresas históricas em sala de aula poderia constituir uma ou mais unidades de ensino em física ou outros cursos, como formação continuada de professores. Como ilustrações, o autor discute as surpresas históricas sobre o trabalho de Isaac Newton sobre difração; experimento do efeito fotoelétrico realizado por Robert A. Millikan; experimentos de espalhamento de raios-X de Arthur H. Comptom; a descoberta do nêutron por James Chadwick; a criação do modelo da gota líquida por George Gamow e a interpretação da fissão nuclear por Lise Meitner e Otto Robert Frisch.

Oon e Subramaniam (2009a, 2009b), relatam o desenvolvimento histórico da natureza da luz desde os tempos dos antigos Gregos até os tempos modernos. Segundo os autores, acompanhar o desenvolvimento do pensamento que caracterizou a evolução dos conceitos na física, ao longo do tempo, permite entender como atualmente compreendemos tais conceitos. Ao longo dos dois trabalhos são destacadas as contribuições dos antigos filosóficos, de Isaac Newton, de Christian Huygens, de Thomas Young, de Augustin Fresnel, de James C. Maxwell, de Max Planck, de Albert Einstein e de Louis de Broglie. No final, os autores comentam sobre possíveis implicações destes relatos para o ensino de Física. 
Klassen (2009) apresenta em forma de narrativa histórica, cinco episódios chaves da história do Efeito fotoelétrico, considerados como necessários para um retrato preciso e adequado: 1) a descoberta do Efeito fotoelétrico por Hertz; 2) a caracterização e a explicação inicial do Efeito fotoelétrico; 3) o artigo revolucionário de Einstein propondo o quantum de luz e a rejeição da comunidade científica à sua ideia de quantização do campo eletromagnético; 4) a verificação experimental de Millikan da equação de Einstein do Efeito fotoelétrico apesar de não aceitar a ideia de quantum de luz de Einstein e 5) a descrição do Efeito Compton utilizando o conceito de quantum de radiação eletromagnética para decisiva aceitação da ideia revolucionária de Einstein. Segundo o autor, a história intitulada "O nascimento do conceito do fóton" foi utilizada/testada quatro vezes com alunos do primeiro do curso de Física, sendo bem recebida todas as vezes. Na prática, segundo o autor, esta ferramenta didática é integrada com um método de instrução que inclui demonstrações experimentais, ilustrações, questionários e exemplo de situações nas quais são aplicados os novos conceitos.

Niaz et al. (2010) enumeraram seis aspectos essenciais de HFC com relação aos eventos que culminaram na proposta da hipótese de quantização da luz de Einstein e na subsequente controvérsia dentro da comunidade científica. Esses aspectos seriam: 1) a hipótese de disparo de Lenard para explicar o Efeito fotoelétrico; 2) a hipótese de quantum de luz de Einstein para explicar o Efeito fotoelétrico; 3 ) o período de rejeição da comunidade científica a ideia de quantum de luz; 4) a determinação experimental de Millikan da equação de Einstein para Efeito fotoelétrico e a determinação empírica do valor da constante de Planck; 5) pré-suposições de Millikan sobre a natureza da luz e 6) apresentação histórica e sua interpretação dentro de uma perspectiva histórica e filosófica da ciência. Utilizando estes aspectos como critérios, Niaz et al. (2010) analisaram 103 livros universitários de Física Geral. Os resultados obtidos revelaram que estes elementos históricos são amplamente ignorados ou distorcidos na maioria dos livros, com apenas três classificados como "satisfatórios" e nenhum como "excelente".

Mangili (2012) utiliza o exemplo do Efeito fotoelétrico para evidenciar alguns nós presentes no estudo da história da Física, mais precisamente nos estudos da História do eletromagnetismo. $\mathrm{O}$ autor busca refletir sobre até que ponto pode-se atribuir a Hertz a descoberta deste efeito. Para isso, ele utiliza várias obras de Hertz que abordam a descoberta do Efeito fotoelétrico bem como estudos relativos ao empreendimento experimental do início do século XX e trabalhos acerca da sociedade alemã neste período.

Todos os trabalhos classificados nessa categoria contribuem substancialmente para enriquecimento das aulas de física, buscando obter a maior quantidade possível de informações sobre a história que envolve a explicação do efeito fotoelétrico, conseguindo assim um relato sem distorções históricas, conceitualmente errôneo ou descontextualizado. 
Também observamos a escassez de trabalhos nacionais no que diz respeito aos aspectos da HFC associados a este tema: encontramos apenas um único trabalho envolvendo HFC ${ }^{3}$. Em nossa busca não identificamos nenhum trabalho investigando a abordagem do efeito fotoelétrico em livros didáticos de Física, especificamente que analisassem a maneira pela qual os livros didáticos abordam a HFC deste tema.

\section{Categoria D: Propostas ou relatos do ensino do efeito fotoelétrico mediados por simulação computacional (11 trabalhos)}

Veit et al. (1987) apresentam um programa-aula via microcomputador para estudantes de ensino médio, no qual realiza-se um confronto entre o modelo corpuscular e o ondulatório para luz. Segundo os autores, o trabalho almejava que os alunos entendessem sequencialmente as relações entre corrente elétrica e diferença de potencial, entre corrente elétrica e intensidade luminosa, entre a energia cinética máxima dos elétrons e frequência da radiação incidente e, por fim, entre frequência limite e o tipo de material utilizado nas placas. Os autores justificam a utilização do microcomputador no ensino deste tema pontuando à pouca tradição didática da área de FMC, a carência de materiais instrucionais como, por exemplo, textos adequados, bem como a impossibilidade de execução de diversas experiências de FMC em sala de aula.

Steinberg et al. (1996) realizaram estudos sobre a aprendizagem do efeito fotoelétrico por estudantes universitários e evidenciaram cinco dificuldades específicas que impedem a aprendizagem significativa deste fenômeno, são elas:

1. [...] A crença de que a Lei de $\mathrm{Ohm}(U=R$. i) aplica-se ao experimento do Efeito fotoelétrico [...]. 2. A incapacidade dos estudantes diferenciarem intensidade de luz de frequência de luz [...]. 3. A crença de que o fóton é um objeto carregado eletricamente [...]. 4. A incapacidade dos estudantes de preverem um gráfico $U$ x i para o experimento do Efeito fotoelétrico [...]. 5. A incapacidade dos estudantes explicarem os resultados experimentais utilizando o modelo corpuscular da luz (STEINBERG et al., 1996, p. 05).

Em resposta a essas dificuldades, Oberem e Steinberg (1999) desenvolveram um tutorial computacional denominado Photoelectric Tutor (PT), no qual os próprios estudantes o completariam, em cerca de uma hora, após a aula tradicional. Os autores afirmaram que o tutorial ajudou os estudantes a terem progressos na previsão dos resultados experimentais quando algum parâmetro era alterado, porém $60 \%$ dos estudantes ainda não foram capazes de prever corretamente os resultados experimentais quando a diferença de potencial elétrico na bateria era alterada.

\footnotetext{
${ }^{3}$ MANGILI, A. I. Heinrich Rudolph Hertz e a "descoberta" do efeito fotoelétrico: Um exemplo dos cuidados que devemos ter ao utilizar a história da ciência na sala de aula. História da Ciência e Ensino: Construindo Interfaces, v. 6, 2012. p. 32-48.
}

da Silva, R. S. e Errobidart, N. C. G. 
De Leone e Oberem (2003) realizaram posteriormente estudos no qual aplicaram o tutorial PT, desenvolvido por Oberem e Steinberg (1999), confirmando quase todas as dificuldades já apontadas por Steinberg et al (1996), excetuando a possibilidade de o fóton ser um objeto carregado eletricamente. Além disso, os autores identificaram uma sexta dificuldade específica de aprendizagem: "a falta de conhecimentos básicos dos estudantes acerca do modelo ondulatório da luz com os quais o experimento do efeito fotoelétrico é contrastado" (DE LEONE; OBEREM, 2003, p. 114).

Em outro trabalho, nessa mesma linha de pesquisa, McKagan et al. (2009) relatam o desenvolvimento ao longo de dois anos de uma reforma curricular sobre o ensino do efeito fotoelétrico num curso engenharia. Ela inclui o aperfeiçoamento do tutorial PT, elaborado por Oberem e Steinberg (1999), aulas interativas com instrução aos pares e resolução problemas conceituais e algébricos.

Ao final da aplicação da proposta, os autores pontuaram que os alunos seriam capazes de: (1) prever corretamente os resultados experimentais para o efeito fotoelétrico quando algum parâmetro é alterado e (2) explicar esses resultados utilizando o modelo corpuscular da luz. Os resultados indicaram que $85 \%$ dos alunos conseguiram prever corretamente os resultados experimentais quando era alterado algum parâmetro no fenômeno, inclusive a diferença de potencial da bateria, afirmam, entretanto, que muitos estudantes ainda não foram capazes de estabelecer uma conexão lógica clara entre as observações e o modelo corpuscular da luz.

Cardoso e Dickman (2012) elaboraram uma unidade de ensino potencialmente significativa, baseada no uso de simulação computacional para o ensino do efeito fotoelétrico, a qual foi aplicada numa turma de terceiro ano do ensino médio. Em uma das atividades proposta na sequência, foi utilizada a simulação do efeito fotoelétrico desenvolvida por McKagan et al. (2008). Os resultados da pesquisa sinalizam que os alunos obtiveram um índice de acerto acima de $67 \%$ na maioria das questões propostas no teste de conhecimento final.

Sokolowski (2013) apresenta a estrutura de um processo de ensino fundamentado na aprendizagem indutiva. Neste processo, utiliza-se um questionário fechado para apresentar aos alunos um problema e, mediante o uso informações auxiliares, estes são guiados do processo de solução. Assim, o autor elaborou e desenvolveu uma unidade de ensino na qual utiliza este tipo de método para ensinar o efeito fotoelétrico. As aulas foram ministradas a um grupo de 15 alunos do (high-school), o que corresponde ao nosso último ano do ensino médio, utilizando uma simulação do experimento como mediadora para instrução. Nas considerações finais, o autor afirma que por meio das análises das respostas dos alunos sobre a influência da bateria no circuito fotoelétrico, pode-se afirmar as aulas atingiram seus objetivos de aprendizagem.

Heckler et al. (2007) relatam o desenvolvimento e a aplicação de um CD-ROM de óptica para o ensino médio. O material contém textos didáticos escritos em linguagem $\mathrm{html}$, animações, simulações (Java Applets) e imagens disponíveis na internet. A proposta foi aplicada em duas turmas de alunos do $3^{\circ}$ ano do ensino médio. $\mathrm{O}$ efeito fotoelétrico e o efeito Compton foram abordados no começo do curso, dentro do tópico "natureza da luz". 
Sales et al (2008) desenvolveram e aplicaram uma atividade de modelagem exploratória, num curso de Física Quântica, oferecido para alunos de Ensino Médio com a utilização do objeto de aprendizagem (OA) denominado Pato Quântico. Neste caso, a metáfora de patos em constante movimento no poleiro quântico representa o efeito fotoelétrico e permite o cálculo da constante de Planck pelos alunos. Os resultados indicam que a interação dos estudantes com o software sinalizou uma aprendizagem significativa do fenômeno, com eficiente exemplo de transposição didática dos saberes e fortalecimento de mudanças conceituais.

Neto et al (2010) apresentam a construção de uma página na Internet, como produto educacional resultante do projeto "A física Moderna no processo de formação de técnicos na área de radiologia médica”. Esta página foi utilizada durante um semestre letivo como material de apoio para a disciplina de "Proteção Radiológica". Durante o ensino do efeito fotoelétrico, os autores destacaram a abordagem interdisciplinar com a física de radiodiagnóstico na utilização de telas intensificadoras (écrans).

Cavalcante et al. (2002) apresentam uma proposta para o ensino do efeito fotoelétrico que envolve dois recursos educacionais distintos: uma simulação computacional e um experimento prático com LED's. Por fim, os autores também indicam uma possível abordagem interdisciplinar entre professores de Física e Filosofia, apontando alternativas para uma prática docente interligada e contextualizada.

Em síntese, os trabalhos relatados nesta categoria relatam experiências de ensino do efeito fotoelétrico, destacando aspectos positivos e negativos, em especial, as principais dificuldades de aprendizagem dos alunos. Vale destacar que a maioria faz uso de roteiro de atividades para utilização do mesmo laboratório virtual, chamado "efeito fotoelétrico",

\section{Categoria E: Propostas ou relatos do ensino do efeito fotoelétrico mediados por HFC (03} artigos)

Pinto e Zanetic (1999) prepararam um minicurso sobre a inserção da natureza quântica da luz, composto de 12 aulas, aplicado nas aulas regulares de física de duas classes do $2^{\circ}$ ano do ensino médio. A noção de perfil epistemológico, de Gaston Bachelard, foi utilizada como referencial filosófico. As aulas abordaram diversas formas do conhecimento físico, entre elas: a descrição histórica da luz, o aspecto filosófico (Perfil Epistemológico) e atividades experimentais. Segundo os autores, a hipótese de que seja possível levar FQ para o Ensino Médio foi confirmada pelo interesse demonstrado por grande parte dos alunos.

Silva e Martins (2009) propõem e relatam uma experiência de um júri simulado no ensino de ótica, especificamente, abordando o tema "natureza da luz", fundamentado pela HFC. Inicialmente os autores justificam o uso de HFC nas aulas de Física no ensino médio como

\footnotetext{
4 Esta simulação, que é parte do Phet Interactive Simulations Project, na Universidade do Colorado. Disponível em: <http://phet.colorado.edu/en/simulation/photoelectric >. Acesso em: 10 nov. 2014.
} 
forma de contribuir para identificação e superação de obstáculos epistemológicos à aprendizagem e possibilidade de dar um maior significado ao estudo desse conteúdo, revelando aspectos histórico-sociais importantes para o desenvolvimento dos conceitos.

Segundo os autores, o papel do professor nesta estratégia é o de coordenador da atividade, porém, antes do dia do júri, este deve prover atividades que fundamentem conceitualmente os alunos sobre o tema. Entre as várias contribuições da estratégia, os autores destacam: favorecimento do trabalho em grupo e o diálogo entre os estudantes; socialização das concepções apresentadas pelos estudantes; incentivo à capacidade de argumentação e elaboração de hipóteses; desenvolvimento da comunicação oral e, por fim, aprendizagem dos conceitos e temas científicos.

Coelho e Borges (2010) relatam um estudo sobre o desenvolvimento do pensamento dos estudantes no campo da óptica e física moderna, especificamente sobre a natureza da luz, em um ambiente curricular no qual os conteúdos são organizados de forma recursiva e em espiral. Os autores investigaram (i) a mudança no entendimento dos estudantes sobre a natureza da luz, ao longo da terceira série do Ensino Médio e (ii) o patamar de entendimento dos estudantes sobre essa temática, ao final da terceira série.

Para avaliar o entendimento dos estudantes, os autores solicitaram que os mesmos respondessem à mesma questão dissertativa (“O que é a luz?”) em duas ocasiões distintas em um intervalo de dez meses. Após a primeira ocasião, foi desenvolvido um sistema categórico hierarquizado constituído de cinco modelos sobre a natureza da luz e, após a segunda ocasião, observaram as possíveis mudanças de categorias dos estudantes.

Os resultados sugeriram que os estudantes possuíam um alto conhecimento prévio e as experiências que eles vivenciaram nesse período produziram efeitos significativos para o progresso nos modelos dos estudantes, mas esses efeitos não foram igualmente verificados em todas as turmas que participaram da análise. Em relação ao patamar de entendimento dos estudantes, os autores apontam que maior parte deles mobiliza os modelos eletromagnético, corpuscular (com a hipótese do quantum de energia) ou dual, mas com alguns estudantes apresentando inconsistências em suas explicações.

Apesar de os trabalhos apresentados nesta categoria buscarem uma abordagem que lança mão de aspectos de HFC, identificamos a inexistência de trabalhos brasileiros que abordam em detalhes a história do efeito fotoelétrico, tal como foi feita nos trabalhos de Kragh (1992), Klassen (2009) e Niaz et al. (2010).

\section{Categoria F: Propostas ou relatos do ensino do efeito fotoelétrico mediados por experi- mentação (08 artigos)}

Arruda e Filho (1991) relatam a utilização de materiais de baixo custo para montar um laboratório de Física. Os autores descrevem diversas experiências, tais como: a produção de altas tensões com a bobina de Tesla, montagem de redes de difração com CD e laser, a utilização de lâmpadas comerciais para a obtenção de espectros e medição do comprimento de 
onda da radiação emitida, a demonstração do efeito fotoelétrico utilizando lâmpadas comerciais de mercúrio. Todas as experiências descritas têm caráter apenas demonstrativo e podem ser utilizadas no ensino fundamental e médio ou para a divulgação científica.

Cavalcante e Tavolaro (2001) desenvolveram uma oficina de FMC que tinha como objetivo sua inserção no ensino médio. Nela discutem especificamente o tópico comportamento dual da luz e da matéria. A natureza ondulatória da luz é demonstrada com a realização de experiências de interferência e difração, utilizando lanternas, cílios postiços e CDs, como instrumentos para a sua decomposição. Já a natureza corpuscular da luz é evidenciada utilizando um "espectrofotômetro caseiro" construído com sensores de calculadoras solares (célula fotoelétrica), LEDs (Light Emitting Diode) e um laser. As autoras também lançam mão da ajuda de simulações computacionais para explicar o efeito fotoelétrico e a difração dos elétrons.

Cavalcante et al. (2005) apresentam diferentes propostas experimentais que permitem estudar espectros de emissão e absorção e ainda uma proposta de baixo custo para a observação de espectro de absorção. Os recursos de baixo custo e a metodologia desenvolvida constituem uma ferramenta tecnológica acessível para identificação das radiações no estudo dos mais variados tipos de estrutura, de gases a sólidos incandescentes. Atenção especial foi dedicada aos semicondutores, mostrando como LEDs comerciais podem substituir tanto as fontes como os sensores de luz na incansável e importante tarefa de verificar a validade de modelos através da determinação de constantes físicas: o modelo do quantum de luz e a constante de Planck.

Filho et al. (2006) relatam o desenvolvimento e a caracterização de uma célula de efeito fotoelétrico e um sistema de excitação e de medição adequados para uso didático em aulas de Física. A célula é semelhante às primeiras desenvolvidas no século XIX, e o sistema de medição envolve transistores, uma bateria e um multímetro, e permite estimar a corrente fotoelétrica produzida na célula. Tal proposta vem a amenizar a inexistência de células fotoelétricas comerciais disponíveis.

Paranhos et al. (2008) realizaram um projeto para o ensino de FMC em um curso de licenciatura em física. Este projeto consistiu no desenho, construção e utilização de um experimento utilizando lâmpada de vapor de mercúrio para atividades práticas e demonstrações didáticas. Os autores propuseram utilizar o experimento para trabalhar os seguintes tópicos: o efeito fotoelétrico, a análise do espectro de emissão do mercúrio e suas particularidades relacionadas às regras de seleção e suas evidências de correções relativísticas.

Silva e Assis (2012) relatam a utilização de uma atividade experimental qualitativa de demonstração construída com materiais de baixo custo para o ensino do efeito fotoelétrico, denominada "Ouça seu controle remoto". Busca-se nesta atividade contextualizar o ensino com algumas aplicações tecnológicas vivenciadas pelos alunos no seu cotidiano a fim de permitir o desencadeamento e a sustentação das interações sociais no ambiente de sala de aula.

Cavalcante et al. $(2013 ; 2014)$ elaboraram uma sequência didática com o objetivo de explorar o funcionamento de controles remoto a partir da utilização do micro controlador Arduino. Como pano de fundo para o trabalho, os autores utilizam o ensino do efeito fotoelétrico. Por ser um tema amplo, dividiu-se a apresentação em duas partes. No primeiro artigo, os autores 
apresentam características mais gerais e mostram como se pode tornar perceptível a radiação infravermelha, quer transformando-a em radiação visível através dos fotossensores presentes em câmeras digitais, quer transformando-a em sinais elétricos que podem ser visualizados através do software de som Audacity disponível livremente na web. No segundo, apresentam uma sequência didática com o objetivo de explorar o funcionamento de controles remoto. Também são propostos experimentos de forma a tornar possível, a compreensão não apenas do seu funcionamento, mas como se processa os diferentes comandos advindos de cada uma de suas teclas.

Em resumo, os trabalhos descritos nesta categoria relatam sugestões para construção de aparatos experimentais de baixo custo, os quais permitiriam ao professor fazer a demonstração do efeito fotoelétrico em sala de aula. Em sua maioria, estes aparatos fazem uso de um resistor dependente da intensidade luminosa - LDR e dos princípios físicos aplicados ao funcionamento de controles remotos.

\section{Categoria G: Análise de livros didáticos ou manuais com relação à abordagem do efeito fotoelétrico (02 artigos)}

Klassen et al. (2011) fizeram a análise de 38 manuais de laboratório relacionado ao efeito fotoelétrico, produzidos para estudantes de graduação em física do ponto de vista pedagógico. $\mathrm{O}$ foco do estudo é a forma como as bases históricas do efeito fotoelétrico são retratadas nestes manuais. Os critérios de avaliação dos manuais são baseados na reconstrução histórica e filosófica do Efeito fotoelétrico, os quais foram apontados no trabalho de Klassen (2009). Como resultado, os autores apontam que nenhum dos manuais foi considerado excelente e que, em geral, ignoram o contexto histórico e dados experimentais que podem levar a interpretações alternativas do efeito. Os autores sugerem fortemente o uso de abordagem histórica para lidar com as dificuldades de compreensão conceitual do efeito fotoelétrico, o que não é facilitado, em investigações laboratoriais.

Alemany et al. (2013) realizaram uma investigação acerca dos aspectos de História da Ciência (HC), especificamente sobre a introdução do conceito de "quantum de luz" na comunidade científica, presentes nos livros didáticos utilizados no $2^{\circ}$ ano do curso de Bacharel em Física, na Universidade de Alicante (Espanha). Em resumo, os autores apontam que o ensino tradicional introduz a descontinuidade da energia da radiação no momento em que são apresentadas as contradições das previsões da teoria eletromagnética com os resultados experimentais do Efeito fotoelétrico.

Os trabalhos relatados nesta categoria apontam para uma necessária análise dos livros didáticos aprovados pelo Plano Nacional do Livro de Didático (2012) levando-se em conta elementos de HFC, visto que não encontramos nenhum artigo que tivesse este enfoque.

A fim de demonstrar de forma mais resumida a classificação dos trabalhos analisados, construímos o gráfico 1, ilustrando o percentual de trabalhos encontrados em cada categoria. 


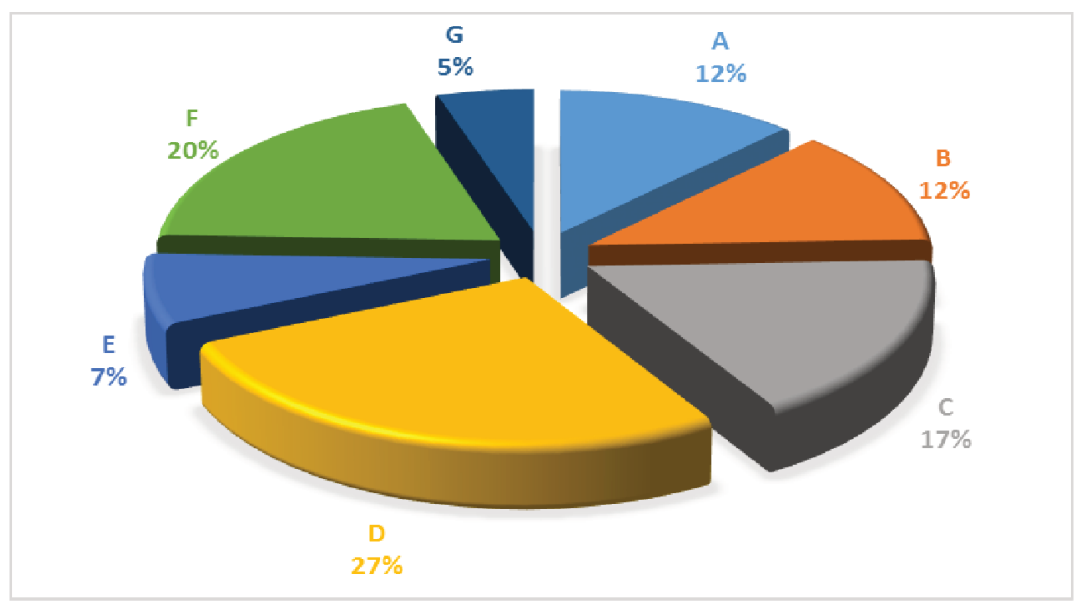

Gráfico 1 - Percentual de trabalhos encontrados por categoria.

Primeiramente, observa-se que $27 \%$ dos trabalhos encontrados propõe a abordagem do efeito fotoelétrico em sala de aula por meio da utilização de simulação computacional (categoria D). Vale destacar novamente que a maioria destes trabalhos fizeram uso de roteiro de atividades para utilização do mesmo laboratório virtual, chamado "efeito fotoelétrico". Em segundo lugar, podemos notar uma concentração de $20 \%$ dos trabalhos propondo construção de aparatos experimentais para demonstração em sala de aula (categoria F), nesta situação, ressaltamos que estes trabalhos foram encontrados somente em periódicos nacionais. Em terceiro lugar, destacamos que um total de $17 \%$ dos trabalhos estavam relacionados aos aspectos de HFC do efeito fotoelétrico (categoria C), neste último caso, a maioria dos trabalhos são internacionais. Estas três categorias concentram, em conjunto, $64 \%$ dos trabalhos encontrados.

Por fim, observamos uma distribuição dos $36 \%$ restante dos trabalhos entre as categorias A, B, E e G, com um pequeno aumento na concentração de trabalhos nas categorias A e B.

\section{Considerações finais}

No presente trabalho, fizemos um levantamento de 41 artigos científicos sobre o ensino do efeito fotoelétrico publicados em revistas nacionais e internacionais, no período de 1987 a 2014 e, em seguida, os classificamos em oito categorias (A), (B), .., (G), levando-se em consideração o enfoque adotado pelos autores. A distribuição do número de trabalhos por categoria possibilitou inferir que maioria deles relata propostas a serem utilizadas em sala de aula mediante utilização de simulação computacional ou experimentação ou aspectos de História e Filosofia da Ciência.

Ostermann e Moreira (2000), juntamente com Greca e Moreira (2001) e Ostermann e Pereira (2009), apontaram que a grande maioria das publicações sobre FMC, no início do século

\footnotetext{
5 Esta simulação, que é parte do Phet Interactive Simulations Project, na Universidade do Colorado. Disponível em: <http://phet.colorado.edu/en/simulation/photoelectric>. Acesso em: 10 nov. 2014.
}

da Silva, R. S. e Errobidart, N. C. G. 
XXI, era em forma de divulgação ou como bibliografia de consulta para professores. Nossa pesquisa permite evidenciar que, em relação ao ensino do efeito fotoelétrico, houve uma notável redução de artigos científicos com esse enfoque. No entanto, corroboramos os apontamentos dos autores de que ainda persiste escassez de trabalhos que investigam efetivamente a construção de conceitos físicos pelos os alunos com relação a este tema. Em nossa pesquisa, evidenciamos que somente cerca de $27 \%$ (onze de quarenta e um) dos trabalhos encontrados se propuseram a investigar os resultados de aprendizagem ${ }^{6}$, indicando uma necessidade maior de investigação sobre o processo de construção dos conceitos científicos presentes no modelo corpuscular para a luz.

Em relação ao uso das relações entre Ciência, Tecnologia e Sociedade (CTS) em sala de aula, nossa pesquisa evidenciou que quase sempre os autores utilizam as aplicações tecnológicas do efeito fotoelétrico como forma de contextualização do conteúdo. Os dispositivos tecnológicos utilizados por esses autores frequentemente são: sistemas automáticos que utilizam um resistor dependente de intensidade luminosa (LDR); células fotovoltaicas e o controle remoto.

Nossa pesquisa também identificou dois trabalhos estrangeiros (KLASSEN, 2009; NIAZ et al., 2010) que optaram por uma abordagem histórica do efeito fotoelétrico em sala de aula. Em síntese, estes autores apontaram que a inserção de elementos da HFC dentro de um método mais amplo de instrução, que inclui também outras ferramentas de ensino, tais como demonstrações experimentais e resolução de problemas, tem possibilitado mostrar que as descobertas científicas são desorganizadas e que não surgem numa sequência simples e ordenada, podendo prevalecer por algumas vezes, num curto prazo, a teoria rival. Esta descrição difere substancialmente daquela que frequentemente é estereotipada nos livros didáticos, pois ressalta a possibilidade de interpretações divergentes para o mesmo fenômeno físico.

Em relação às pesquisas que propuseram uma abordagem em sala de aula por meio da utilização de simulação computacional, observamos que, em geral, os resultados de aprendizagem apontados pelos autores indicam que a maioria dos alunos conseguiu prever corretamente os resultados experimentais quando é alterado algum parâmetro na simulação, porém, em contrapartida, a maioria também tem dificuldades em estabelecer uma conexão lógica e clara entre as observações experimentais e o modelo corpuscular da luz.

Por fim, nosso trabalho permitiu identificar quatro possíveis dificuldades dos alunos de nível médio quanto à aprendizagem do modelo corpuscular da luz, que são mais recorrentes na literatura: (1) a falta de diferenciação dos conceitos de intensidade e frequência de luz; (2) a falta de compreensão do conceito de função trabalho de uma superfície metálica; (3) a falta conhecimentos básicos dos estudantes acerca do modelo ondulatório da luz, com os quais o

\footnotetext{
6 STEINBERG et al., 1996; OBEREM; STEINBERG, 1999; PINTO; ZANETIC, 1999; DE LEONE; OBEREM, 2003; HECKLER et al., 2007; SALES et al., 2008; KLASSEN, 2009; MCKAGAN et al., 2009; COELHO; BORGES, 2010; CARDOSO; DICKMAN, 2012; SOKOLOWSKI, 2013.
} 
experimento do efeito fotoelétrico é contrastado e (4) a falta de domínio de operações básicas de matemática para resolução de problemas.

Os dados obtidos nessa investigação foram utilizados para estruturar uma unidade de ensino potencialmente significativa, principalmente as orientações quanto ao emprego de História da Ciência, buscando evitar narrativas linearizadas; relatos romantizados; desprezo do erro; interpretação única das evidências experimentais e aceitação imediata de novas ideias perante comprovação experimental, e simulação computacional.

Os trabalhos de Kragh (1992), Klassen (2009) e Niaz et al. (2010) foram utilizados para estruturar a abordagem da história da nossa unidade de ensino por meio de seis episódios: a) a descoberta do Efeito Fotoelétrico por Hertz; b) a hipótese de disparo de Lenard para explicar o Efeito Fotoelétrico; c) a hipótese rival de quantum de luz de Einstein para explicar o Efeito Fotoelétrico; d) o período de rejeição da comunidade científica a ideia de quantum de luz; e) a verificação experimental de Millikan da equação de Einstein do Efeito Fotoelétrico apesar de não aceitar a ideia de quantum de luz de Einstein e g) a descrição do Efeito Compton utilizando o conceito de quantum de radiação eletromagnética para decisiva aceitação da ideia revolucionária de Einstein.

A simulação computacional foi empregada com o objetivo de ampliar o engajamento dos sujeitos nas ações proposta para a compreensão do efeito fotoelétrico, aspectos pontuados principalmente por Cardoso e Dickman (2012): a testagem de hipóteses; a obtenção de uma postura mais participativa dos alunos; a observação em outra perspectiva de um fenômeno abstrato.

Os resultados de aprendizagem obtidos com a aplicação de nossa unidade de ensino possivelmente serão divulgados em breve.

\section{Referências}

ALEMANY, F. S; BLANCO, J. L. D.; TORREGROSA, J. M. La introducción del concepto de fotón en bachillerato. Revista Brasileira de Ensino de Física, v. 35, n. 2, abr. 2013.

ALVES, E. G.; SILVA, A. F. Usando um LED como fonte de energia. A Física na Escola, v. 9, n. 1, dez. 2008.

ARRUDA, S. M.; FILHO, D. O. T. Laboratório Caseiro de Física Moderna. Caderno Brasileiro de Ensino de Física, v. 8, n. 3, dez. 1991.

CARDOSO, S. O. O.; DICKMAN, A. G. Simulação computacional aliada à teoria da aprendizagem significativa: uma ferramenta para o ensino e aprendizagem do Efeito Fotoelétrico. Caderno Brasileiro de Ensino de Física, v. 29, n. Especial 2, p. 891-934, out. 2012.

CAVAlCAnTE, M. A.; RODRIGUES, T. T.T.; BUENO, D. A. Controle Remoto: Princípio de funcionamento (parte 1 de 2). Caderno Brasileiro de Ensino de física, v. 30, n. 3, p. 554565, dez. 2013.

da Silva, R. S. e Errobidart, N. C. G. 
. Controle Remoto: observando códigos com o Arduíno (parte 2 de 2).

Caderno Brasileiro de Ensino de Física, v. 31, n. 3, p. 614-641, dez. 2014.

CAVAlCANTE, M. A.; TAVOLARO, C. R. C. Uma oficina de Física Moderna que visa sua inserção no ensino médio. Caderno Catarinense de Ensino de Física, Florianópolis, v. 18, n. 3, p. 263-276, dez. 2001.

CAVAlCANTE, M. A.; TAVOLARO, C. R. C.; HAAG, R. Experiências em Física Moderna. A Física na Escola, v. 6, n. 1, 2005.

CAVAlCANTE, M. A.; TAVOLARO, C. R. C; SOUZA, D. F.; MUZINATTI, J. Uma Aula sobre o efeito fotoelétrico no desenvolvimento de competências e habilidades. A Física na Escola, São Paulo, v. 3, n. 1, p. 24-29, maio 2002.

COELHO, G. R.; BORGES, O. O entendimento dos estudantes sobre a natureza da luz em um currículo recursivo. Caderno Brasileiro de Ensino de Física, v. 27, n. 1, p. 63-87, abr. 2010.

DE LEONE, C. J; OBEREM, G. E. Physics Education Research Conference Proceedings. American Institute of Physics, Melville, New York, 2003.

DIONÍSIO, P. H. Albert Einstein e a Física Quântica. Caderno Brasileiro de Ensino de Física, v. 22 , n. 2, p. 147-164, ago. 2005.

FILHO, J. B. R.; SALAMI, M. A.; HILLEBRAND, V. Construção e caracterização de uma célula fotoelétrica para fins didáticos. Revista Brasileira de Ensino de Física, v. 28, n. 4, p. 555-561, ago. 2006.

GOMES, P. R. S.; MORAES, S. B.; SANTOS, M. S.; COSTA, I.; ALMEIDA, L. Aplicação da Espectroscopia Gama no Ensino de Física Moderna. Revista Brasileira de Ensino de Física, v. 18, n. 4, dez. 1996.

GRECA, I. M; MOREIRA, M. A. Uma revisão de literatura sobre estudos relativos ao ensino da Mecânica Quântica introdutória. Investigações em Ensino de Ciências, Porto Alegre, v. 6, n. 1, p. 29-56. 2001.

GUNEL, M.; HAND, B.; GUNDUZ, S. Comparing student understanding of quantum physics when embedding multimodal representations into two different writing format: presentation format versus summary report format. Science Education, Hoboken, v. 90, n. 6, p. 1092-1112, nov. 2006.

HECKLER, V.; SARAIVA, M. F. O.; FILHO, K. S. O. Uso de simuladores, imagens e animações como ferramentas auxiliares no ensino/aprendizagem de óptica. Revista Brasileira de Ensino de Física, v. 29, n. 2, p. 267-273, jul. 2007. 
JONES, D. G. C.Teaching modern physics -microconceptions of the photon that can damage understanding. Physics Education, v. 26, n. 2, p. 93-98, mar. 1991.

KLASSEN S. The Photoelectric Effect: Reconstructing the Story for the Physics Classroom. Science \& Education, v. 20, p. 719-731, nov. 2009.

KLASSEN, S.; NIAZ, M.; METZ, D.; McMILLAN, B.; DIETRICH, S. Portrayal of the History of the Photoelectric Effect in Laboratory Instructions. Science \& Education, v. 21, p. 729-743, may. 2011.

KOVAČEVIĆ, M. S.; DJORDJEVICH, A. A mechanical analogy for the photoelectric effect. Physics Education, v. 41, n. 6, p. 551-555, nov. 2006.

KRAGH, H. A sense of history: History of science and the teaching of introductory quantum theory. Science \& Education, v. 1, p. 349-363, jan. 1992.

MANGILI, A. I. Heinrich Rudolph Hertz e a "descoberta" do efeito fotoelétrico: Um exemplo dos cuidados que devemos ter ao utilizar a história da ciência na sala de aula. História da Ciência e Ensino: Construindo Interfaces, v. 6, p. 32-48, 2012

McKAGAN, S. B.; HANDLEY, W.; WIEMAN, C. E. A Research-Based Curriculum for Teaching the Photoelectric Effect. American Jounal of Physics, v. 77, p. 87-94, jun. 2009.

MEDEIROS, A. Dos mistérios da Física Clássica ao nascimento da Teoria Quântica. A Física na Escola, v. 6, n. 1, abr. 2005.

MOREIRA, I. C. 1905: Um ano miraculoso. A Física na Escola, v. 6, n. 1, dez. 2005.

NETO, J. S.; OSTERMANN, F.; PRADO, S. D. Formação de técnicos na área de radiologia médica: Desenvolvimento de uma página da internet como recurso didático. A Física na Escola, v. 11, n. 1, abr. 2010.

NIAZ, M.; KLASSEN, S.; McMILLAN, B.; METZ, D. Reconstruction of the history of the photoelectric effect and its implications of general physics textbooks. Science \& Education, v. 94, n. 5, p. 903-931, sep. 2010.

OBEREM, G. E.; STEINBERG, R. N. Photolectric Tutor. Physics Academic Software, American Institute of Physics, College Park, MD, 1999.

OON, P. T.; SUBRAMANIAM, R. The nature of light: I. A historical survey up to the prePlanck era and implications for teaching. Physics Education, v. 44, n. 4, p. 384-391,jul. 2009.

The nature of light: II. A historical survey from the Planck era and implications for budding physicists. Physics Education, v. 44, n. 4, p. 392-397, jul. 2009. 
OSTERMANN, F.; MOREIRA, M. A. Uma revisão bibliográfica sobre a área de pesquisa "Física Moderna e Contemporânea no Ensino Médio". Investigações em Ensino de Ciências, Porto Alegre, v. 5, n. 1, p. 23-48, jan. 2000.

OSTERMANN, F.; PEREIRA, A. P. Sobre o ensino de Física Moderna e Contemporânea: uma revisão da produção acadêmica recente. Investigações em ensino de Ciências, v. 14, n 3, p393-420. 2009.

PARANHOS, R. R. G.; R, V. L.; PIZANI, P. S. Lâmpada de Hg para experimentos e demonstrações de física moderna: introdução ao Efeito Fotoelétrico e outros tópicos. Revista Brasileira de Ensino de Física, v. 30, n. 4, p. 4502/0-4502/6, ago. 2008.

PINTO, A.C.; ZANETIC, J. É possível levar a Física Quântica para o ensino médio? Caderno Catarinense de Ensino de Física, Florianópolis, v. 16, n. 1, p. 7-34, abr. 1999.

ROSA, P. R. da S. Uma introdução à pesquisa qualitativa em ensino. 1. ed. Campo Grande: Editora UFMS, 2013. v. 1. 167 p.

SAlES, G. L.; VASCONCELOS, F. H. L.; FILHO, J. A. C.; PEQUENO, M. C. Atividades de modelagem exploratória aplicada ao ensino de física moderna com a utilização do objeto de aprendizagem pato quântico. Revista Brasileira de Ensino de Física, v. 30, n. 3, p. 3501/03501/13, ago. 2008.

SILVA, B. V. C.; MARTINS, A. F. P. Júri simulado: um uso da história e filosofia da Ciência no ensino de óptica. A Física na Escola, v. 10, n. 1, 2009.

SILVA, C. J. O efeito fotoelétrico - contribuições ao ensino de física contemporânea no segundo grau. 1993. 154 p. Dissertação (Mestrado em Ensino de Ciências) - Instituto de Física e Faculdade de Educação, USP, São Paulo.

SILVA, L. F.; ASSIS, A. Física Moderna no Ensino Médio: Um experimento para abordar o Efeito Fotoelétrico. Caderno Brasileiro de Ensino de Física, v. 29, n. 2, p. 313-324, ago. 2012.

SOKOLOWSKI, A. Teaching the photoelectric effect inductively. Physics Education, v. 48, n. 1, p. $35-41.2013$.

STEINBERG, R. N.; OBEREM, G. E.; McDERMOTT, L. C. Development of a computerbased tutorial on the photoelectric effect. American Journal of Physics, v. 64, n. 11, p. 13701379. 1996

STUEWER, R. H. Historical Surprises. Science \& Education, v. 15, p. 521-530, 2006. 
VALADARES, E. C.; MOREIRA, A. M. Ensinando física moderna no segundo grau: efeito fotoelétrico, laser e emissão de corpo negro. Caderno Catarinense de Ensino de Física, Florianópolis, v. 15, n. 2, p. 121-135, ago. 1998.

VEIT, E. A.; THOMAS, G.; FRIES, S. G.; AXT, R.; SELISTRE, L. F. O efeito fotoelétrico no $2^{\circ}$ grau via microcomputador. Caderno Catarinense de Ensino de Física, Florianópolis, v. 4, n. 2, p. 68-88, ago. 1987.

WHALLEY, M. The photoelectric effect: a useful sporting analogy. Physics Education, v. 40, n. 1, p. 503-504, nov. 2005.

WILLIANS, G. Photoelectric effect can be memorable without being expensive. Physics Education, v. 39, n. 2, p. 132-134, mar. 2004. 